\title{
The AaCBF4-AaBAM3.1 module enhances freezing tolerance of kiwifruit (Actinidia arguta)
}

\author{
Shihang Sun ${ }^{1,2}$, Chungen $\mathrm{Hu}^{2}$, Xiujuan Qi ${ }^{1}$, Jinyong Chen', Yunpeng Zhong ${ }^{1}$, Abid Muhammad ${ }^{1}$, Miaomiao Lin ${ }^{1}$ and \\ Jinbao Fang ${ }^{1}$
}

\begin{abstract}
Beta-amylase (BAM) plays an important role in plant resistance to cold stress. However, the specific role of the BAM gene in freezing tolerance is poorly understood. In this study, we demonstrated that a cold-responsive gene module was involved in the freezing tolerance of kiwifruit. In this module, the expression of AaBAM3.1, which encodes a functional protein, was induced by cold stress. AaBAM3.1-overexpressing kiwifruit lines showed increased freezing tolerance, and the heterologous overexpression of AaBAM3.1 in Arabidopsis thaliana resulted in a similar phenotype. The results of promoter GUS activity and cis-element analyses predicted AaCBF4 to be an upstream transcription factor that could regulate AaBAM3.1 expression. Further investigation of protein-DNA interactions by using yeast one-hybrid, GUS coexpression, and dual luciferase reporter assays confirmed that AaCBF4 directly regulated AaBAM3.1 expression. In addition, the expression of both AaBAM3.1 and AaCBF4 in kiwifruit responded positively to cold stress. Hence, we conclude that the AaCBF-AaBAM module is involved in the positive regulation of the freezing tolerance of kiwifruit.
\end{abstract}

\section{Introduction}

Cold stress is a kind of abiotic stress that can considerably limit both plant growth and yield and can determine the geographic distribution of plants ${ }^{1}$. Therefore, freezing tolerance acts as an important quantitative trait and has received considerable amounts of attention for the past several decades ${ }^{2-5}$. In model plant species, researchers have identified complex signaling networks consisting of cold sensors, secondary messengers, transcription factors (TFs), phytohormones and functional proteins ${ }^{6-9}$. Low temperature is also a common factor that significantly hinders the quality and productivity of kiwifruit. In the last twenty years, kiwifruit has often suffered from cold damage worldwide, which has had a large influence on the kiwifruit industry. However, there

\footnotetext{
Correspondence: Miaomiao Lin (linmiaomiao@caas.cn) or Jinbao Fang (fangjinbao@caas.cn)

${ }^{1}$ Key Laboratory for Fruit Tree Growth, Development and Quality Control, Zhengzhou Fruit Research Institute, Chinese Academy of Agricultural Sciences, Zhengzhou 450009, China

${ }^{2}$ Key Laboratory of Horticultural Plant Biology (Ministry of Education), College of Horticulture and Forestry Science, Huazhong Agricultural University, Wuhan 430070, China
}

have been few studies on the freezing tolerance of kiwifruit. Hence, there is an urgent need to understand the freezing tolerance mechanisms of kiwifruit under cold stress to develop novel genetic resources affording strong freezing tolerance ${ }^{10}$.

Cold stress can cause two major types of injury: osmotic stress and oxidative stress ${ }^{11,12}$. When plants are subjected to cold stress, their cells ultimately experience osmotic stress due to the formation of ice crystals ${ }^{13}$. Under osmotic stress, the changes in the accumulation of compatible solutes are the most prominent alterations observed among the physiological and biochemical characteristics of plants ${ }^{14}$. Proline and soluble sugars, which act as compatible solutes that are involved in multiple metabolic pathways, are produced and accumulate under cold stress ${ }^{15}$. Oxidative stress causes the excessive accumulation of reactive oxygen species (ROS) due to an imbalance between their production and scavenging ${ }^{16}$. ROS cause the denaturation of lipids, proteins and nucleic acids, which can lead to further metabolic disorders ${ }^{17}$. Both osmotic and oxidative stresses develop simultaneously by causing synergistic effects under cold stress.

\section{(c) The Author(s) 2021}

(c) (i) Open Access This article is licensed under a Creative Commons Attribution 4.0 International License, which permits use, sharing, adaptation, distribution and reproduction cc) in any medium or format, as long as you give appropriate credit to the original author(s) and the source, provide a link to the Creative Commons license, and indicate if changes were made. The images or other third party material in this article are included in the article's Creative Commons license, unless indicated otherwise in a credit line to the material. If material is not included in the article's Creative Commons license and your intended use is not permitted by statutory regulation or exceeds the permitted use, you will need to obtain permission directly from the copyright holder. To view a copy of this license, visit http://creativecommons.org/licenses/by/4.0/. 
Moreover, it is gradually becoming understood that compatible solutes have important effects on ROS scavenging and enhanced freezing tolerance ${ }^{18}$. To sense and adapt to cold stress, plants have developed sophisticated and efficient mechanisms to protect themselves from cold injury, and the expression of a number of genes is induced in response to low temperature to provide resistance to both osmotic stress and oxidative stress ${ }^{19}$.

In the early 1980s, we had a poor understanding of beta-amylases (BAMs), which are considered to be dispensable storage proteins ${ }^{20,21}$. We have now acquired detailed information about BAMs, which have been shown to play key roles in response to abiotic stress by degrading starch $^{22}$. BAMs are members of glycosyl hydrolase family 14 (PF01373) and have two typical catalytic sites at the $\mathrm{N}$-terminus and in a central location; thus, these enzymes can hydrolyze 1,4-alpha-glucosidic linkages within $\operatorname{starch}^{23}$. BAMs are considered to be the mediators that degrade starch into downstream soluble sugars and play a pivotal role in the accumulation of soluble sugars under cold stress. Moreover, soluble sugars derived from the degradation of starch are transferred from chloroplasts to the cytoplasm and can then participate in the energy metabolism pathway to resist cold damage $^{24,25}$. In perennial plants, previous studies have indicated that the expression level of $B A M$ mRNA and the activity of BAMs could be elevated in pear, blueberry, orange and tea trees under cold stress ${ }^{26-29}$. BAM activity has also been shown to increase in potato under low temperature $^{30}$. The idea that $B A M$ is involved in the cold stress response was further verified in poplar, where $C B F 1$ is the upstream regulatory gene of $B A M^{31}$. In Arabidopsis thaliana (A. thaliana), nine BAM family members have been identified in the genome: BAM1 to BAM9 $^{32}$. A thaliana BAMs can be divided into two categories based on whether they show catalytic activity: BAM1-BAM3, BAM5 and BAM6 are catalytically active, whereas BAM4 and BAM7-BAM9 are proposed to be catalytically inactive. Many studies have demonstrated that $B A M 3$ is transcriptionally induced in response to low temperature, which indicates that cold stress might accelerate starch degradation ${ }^{33}$. However, the suppression of StBAM1 expression results in only low BAM activity and freezing tolerance, indicating that StBAM1 plays a small role in starch degradation, although it exhibits BAM catalytic activity ${ }^{34}$. In addition, the AtBAM3 gene orthologs of various plant species are responsible for soluble sugar accumulation and enhanced freezing tolerance. These findings show that within the plant genome, AtBAM3 and its orthologous genes exhibit the same function in the enhancement of freezing tolerance by degrading starch. Although the BAMs of A. thaliana have been characterized, their functions in freezing tolerance remain mostly unknown. Moreover, the available information about the functions of $B A M$ s in perennial plants is still inadequate.

Over the past few decades, increasing amounts of attention have been focused on TFs. Among TFs, those encoded by C-repeat-binding factor $(C B F)$ genes are thought to be mainly involved in the response to cold stress $^{35,36}$. Furthermore, the CBF cold response pathway has been indicated to play a key role in the enhancement of freezing tolerance. Among the cold-responsive genes of A. thaliana, $12 \%$ can be assigned to the CBF regulon ${ }^{37}$. $A t C B F 1, A t C B F 2$, and AtCBF3 (also referred to as AtDREB1b, AtDREB1c, and AtDREB1a, respectively) have been functionally identified in $A$. thaliana ${ }^{38}$. The mRNA expression of $A t C B F 1, A t C B F 2$, and $A t C B F 3$ has been reported to be quickly induced under cold stress, after which the encoded proteins recognize C-repeat/dehydration-responsive (CRT/DRE) cis-elements in the promoters of COLD-REGULATED (COR) genes and bind to these motifs. It has been determined that $C B F$ expression induces the expression of a large number of COR genes, which results in constitutively enhanced freezing tolerance $^{39}$. In contrast, the silencing of the AtCBF1, AtCBF2, and $A t C B F 3$ genes decreases the mRNA expression of COR genes with CRT/DRE elements, which leads to weakened freezing tolerance of plants ${ }^{40}$. Taken together, the above findings suggest that $C B F$ genes are involved in extensive cold regulatory networks and play an important role in the response to cold stress.

The Actinidia genus includes 54 species and 21 varieties, all of which have varying freezing tolerance abilities $^{41,42}$. Both Actinidia chinensis and Actinidia deliciosa are currently extensively cultivated worldwide, but these two species show very poor tolerance to $-13^{\circ} \mathrm{C}$ temperatures during winter, which causes severe damage to plants $^{43}$. Actinidia. arguta, a prominent species within Actinidia, can survive at a temperature of $-40{ }^{\circ} \mathrm{C}^{42}$. The variable freezing tolerance abilities within the Actinidia genus imply that large genetic variations exist in the kiwifruit genome. Therefore, the identification of coldresponsive genes may allow the detailed molecular mechanisms underlying the enhanced freezing tolerance abilities of kiwifruit to be further explored for molecular breeding purposes.

In this work, we identified the A. arguta AaBAM3.1 gene, which was significantly expressed in response to cold treatment. AaBAM3.1-overexpressing lines were generated in both kiwifruit ("Hongyang") and A. thaliana, and the freezing tolerance of the transgenic plants was enhanced. The promoter region of AaBAM3.1 in transgenic $A$. thaliana and its predicted cis-elements also responded to cold treatment, which indicated that $\mathrm{AaCBF} 4$ may be a candidate for the upstream regulation of $A a B A M 3.1$. Through protein-DNA interaction assays, we found that AaCBF4 directly regulated AaBAM3.1 in 
kiwifruit. Hence, we concluded that kiwifruit AaBAM3.1, which is positively regulated by $\mathrm{AaCBF} 4$, is a coldresponsive gene that can enhance freezing tolerance.

\section{Results}

\section{Cloning of AaBAM3.1 and its sequence analysis}

In a previous study, we cloned and identified a coldinduced BAM gene (GenBank Accession No. MT263012) from A. arguta. AaBAM3.1 had a full-length ORF of 1644 bp flanking a 1363 bp promoter ${ }^{23}$. AaBAM3.1 encoded a protein of 548 amino acids with a $61.74 \mathrm{kDa}$ molecular weight, and the theoretical isoelectric point of AaBAM3.1 was 8.76. A maximum likelihood tree was established using 14 BAM protein sequences. The BAM sequences from different plant species clustered into two main groups, I and II. AaBAM3.1 was classified into the same group (part 'I', corresponding to the red region in the Fig. S4a) as AtBAM3 of A. thaliana (Fig. S4a). Multiple alignment revealed a highly conserved catalytic and binding site among AaBAM3.1 and 9 other BAM proteins (Fig. S4b). The conserved site contained 2 catalytic residues, Glu186 and Glu380 (black arrowheads in the Fig. S4b), and 19 substrate-binding residues (red arrowheads in the Fig. S4b).

\section{Time course of AaBAM3.1 expression in A. arguta under cold stress}

Thirty-day-old $A$. arguta plants were cold subjected to $4{ }^{\circ} \mathrm{C}$ for various times. Imaging analysis demonstrated that chlorophyll fluorescence was lower in the leaves of the treated kiwifruit than in those of the untreated kiwifruit (Fig. 1a). Accordingly, the $\mathrm{Fv} / \mathrm{Fm}$ of the leaves was progressively reduced by cold stress (Fig. 1b). The observed levels of relative electrolyte leakage (REL), a major indicator of membrane damage, indicated that membrane damage was significantly greater in the treated kiwifruit than in the untreated kiwifruit (Fig. 1c). The transcript level of AaBAM3.1 gradually increased over 5 days of cold treatment (Fig. 1d). The results of tissue-specific expression analysis showed the highest expression level in the leaves during the growth stage (Fig. 1e), while the expression level in the shoots was lowest and increased in the dormant stage. When the air temperature decreased to the lowest level at the dormant stage, the expression level of AaBAM3.1 reached the highest level (Fig. 1f). Taken together, the results indicated that $A a B A M 3.1 \mathrm{can}$ respond to cold stress.

\section{In vitro analysis of the subcellular localization of the AaBAM3.1 protein and BAM enzyme activity}

The subcellular localization of AaBAM3.1 was examined via its transient expression in tobacco. We constructed an AaBAM3.1-green fluorescent protein (GFP) fusion vector and used the empty vector (EV) containing
GFP alone as a control. These plasmids, driven by the CaMV $35 \mathrm{~S}$ promoter, were injected into the tobacco leaf epidermis using the Agrobacterium-mediated transient expression method. GFP fluorescence was ubiquitously distributed around the whole cell in those containing the control plasmid (Fig. 2a). By contrast, the GFP signal in cells containing the recombinant plasmid was observed solely in the cytoplasm and overlapped with the red autofluorescence of chloroplasts. Overall, the results indicated that AaBAM3.1 was located in the chloroplast.

Overexpression of the AaBAM3.1-glutathione S-transferase (GST) fusion plasmid in E. coli Rosetta (DE3) cells was induced by IPTG. The total purified proteins and the induced soluble protein fraction were isolated via SDSPAGE, and AaBAM3.1 was shown to be successfully expressed (Fig. 2b). The proteins whose expression was induced were used to analyze enzyme activity. The results indicated that the fusion protein could catalyze the transformation of starch into maltose. As expected, the control $\left(\mathrm{ddH}_{2} \mathrm{O}\right)$ exhibited no obvious BAM activity.

\section{Heterologous expression of AaBAM3.1 enhances cold tolerance of $A$. thaliana}

The transgenic and wild-type plants did not show obvious phenotypic differences under normal growth conditions. When 3-week-old plants were subjected to $-2{ }^{\circ} \mathrm{C}$ for $2 \mathrm{~h}$, the WT plants showed more severe cold injury than did the transgenic plants (Fig. 3a). After freezing stress, the average survival rate of the AaBAM3.1-overexpressing plants was higher (>40\%) than that of the WT plants (<20\%) (Fig. 3d). Malondialdehyde (MDA) and REL levels were measured to assess membrane damage in the treated plants. We observed significantly lower levels of MDA and REL in the transgenic plants than in the WT plants under cold stress conditions (Fig. 3e, h). Previous studies have shown that the activities of antioxidant enzymes such as superoxide dismutase (SOD), peroxidase (POD), and catalase (CAT) play an important role in enhancing freezing tolerance. We also observed that the transgenic plants exhibited significantly higher SOD, POD, and CAT activities than the WT plants did under the $-2{ }^{\circ} \mathrm{C}$ treatment (Fig. 3j, k, l). Proline (PRO) and soluble sugars (SS) are considered important compatible solutes that help plants cope with abiotic stress. After $-2{ }^{\circ} \mathrm{C}$ treatment, the PRO and SS contents in the transgenic plants were significantly higher than those in the WT plants (Fig. 3f, g). Under the $-2{ }^{\circ} \mathrm{C}$ treatment, in situ histochemical staining via diaminobenzidine (DAB) and nitro blue tetrazolium (NBT) revealed that $\mathrm{H}_{2} \mathrm{O}_{2}$ and $\mathrm{O}_{2}{ }^{-}$accumulated more in the WT plants than in the transgenic plants (Fig. 3b). Chlorophyll fluorescence imaging demonstrated that the leaves of the transgenic plants maintained a higher level of chlorophyll fluorescence than did those of the WT plants under cold 


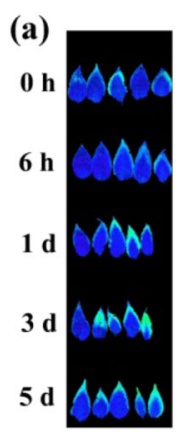

(b)

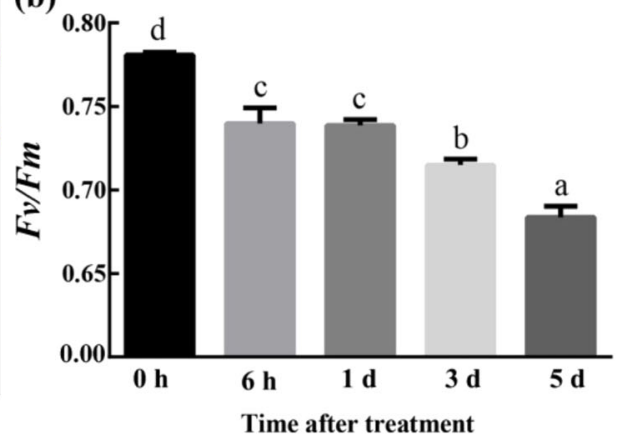

(e)

(d)

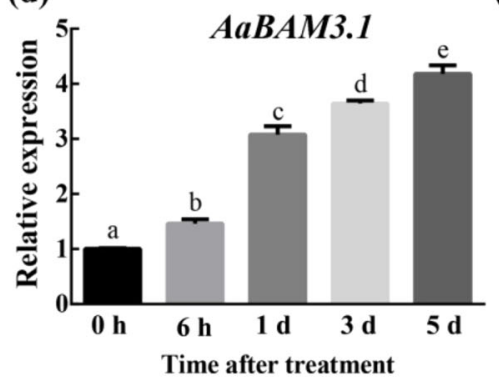

(c)

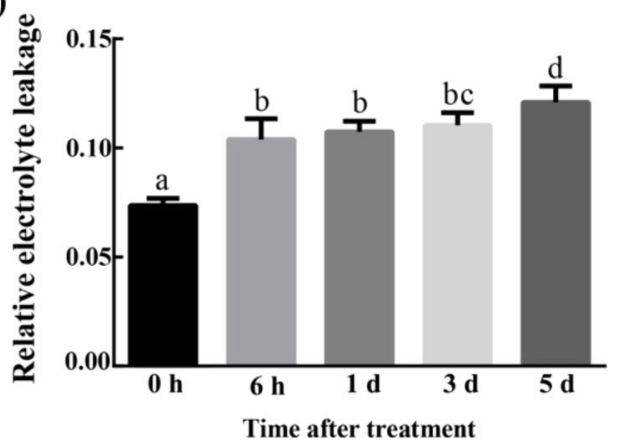

(f)

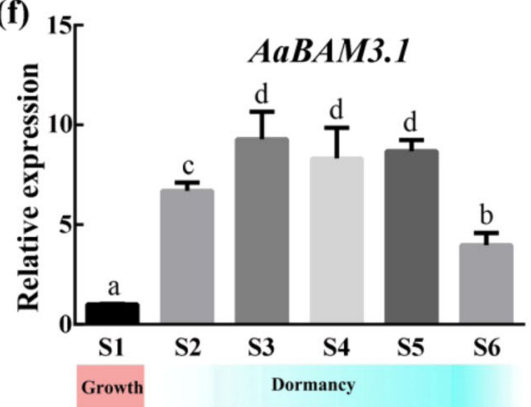

Fig. 1 Phenotypes of Actinidia arguta plants under cold stress and the expression of AaBAM3.1 in A. arguta in response to cold stress. a Chlorophyll fluorescence image. $\mathbf{b}$ Corresponding $F_{v} / F_{m}$ over the course of a 5-day low-temperature treatment. $\mathbf{c}$ Electrolyte leakage of Actinidia arguta plants after being subjected to 5 days of low-temperature treatment. $\mathbf{d}$ The time course of expression levels in the leaves was analyzed via RTqPCR in response to cold stress. e Tissue-specific expression in the vegetative growth stage was analyzed via RT-qPCR. f AaBAM3.1 expression in the shoots was analyzed between the growth stage and dormant stage under cold stress. S1: 15 April; S2: 15 November; S3: 7 December; S4: 23 December; S5: 15 January; S6: 15 March

stress. The $F v / F m$ values of both the transgenic and WT plants were reduced under cold stress; however, the transgenic plants displayed higher $F v / F m$ values than did the WT plants (Fig. 3c, m). After cold stress, the relative expression of AaBAM3.1 was higher in the treated transgenic kiwifruit plants than in the untreated transgenic plants (Fig. 3n). Taken together, the above results indicated that AaBAM3.1 contributes to freezing tolerance by decreasing the ROS, REL and MDA levels and increasing the antioxidant enzyme activities and contents of compatible solutes.

\section{AaBAM3.1 overexpression in $A$. chinensis enhances cold tolerance}

To confirm the roles of $A a B A M 3.1$ in kiwifruit, we obtained AaBAM3.1-overexpressing plants of $A$. chinensis $\mathrm{cv}$. Hongyang by transforming explants produced from leaf strips. Three lines with high expression of AaBAM3.1 were chosen for further studies. The cold tolerance of transgenic kiwifruit was assessed by analyzing the REL levels and $F v / F m$, taking WT plants as controls. After subjecting 30-day-old plants to $2{ }^{\circ} \mathrm{C}$ treatment for $1 \mathrm{~h}$, the WT plants showed more severe cold injury than did the transgenic plants (Fig. 4a). Under low-temperature stress, the REL and MDA levels in the transgenic plants were significantly lower than those in the WT plants (Fig. 4d, e). In addition, the $F v / F m$ was lower in the transgenic lines than in the WT (Fig. 4c, i). DAB and NBT staining indicated that the transgenic plants accumulated less $\mathrm{H}_{2} \mathrm{O}_{2}$ and $\mathrm{O}_{2}{ }^{-}$than did the WT plants. Moreover, the levels of ROS were significantly lower in the transgenic plants than in the WT plants under cold stress (Fig. 4b). Beta-amylase activity was significantly higher in the A. chinensis transgenic lines than in the the WT plants after cold stress (Fig. 4g). In addition, SS levels were higher in the transgenic lines than in WT plants (Fig. 4f). After treatment, the relative expression of AaBAM3.1 was higher in the treated transgenic plants than in the untreated transgenic plants (Fig. 4h). From the above results, we can infer the positive involvement of AaBAM3.1 in enhancing the cold tolerance of kiwifruit.

\section{Promoter element analysis}

We used the NewPLACE database and conducted a manual search to identify the cis-elements in the AaBAM3.1 promoter sequences $(\sim 1.3 \mathrm{~kb})$ that were involved in the plant cold response to reveal the transcriptional regulation network of the AaBAM3.1 gene. 

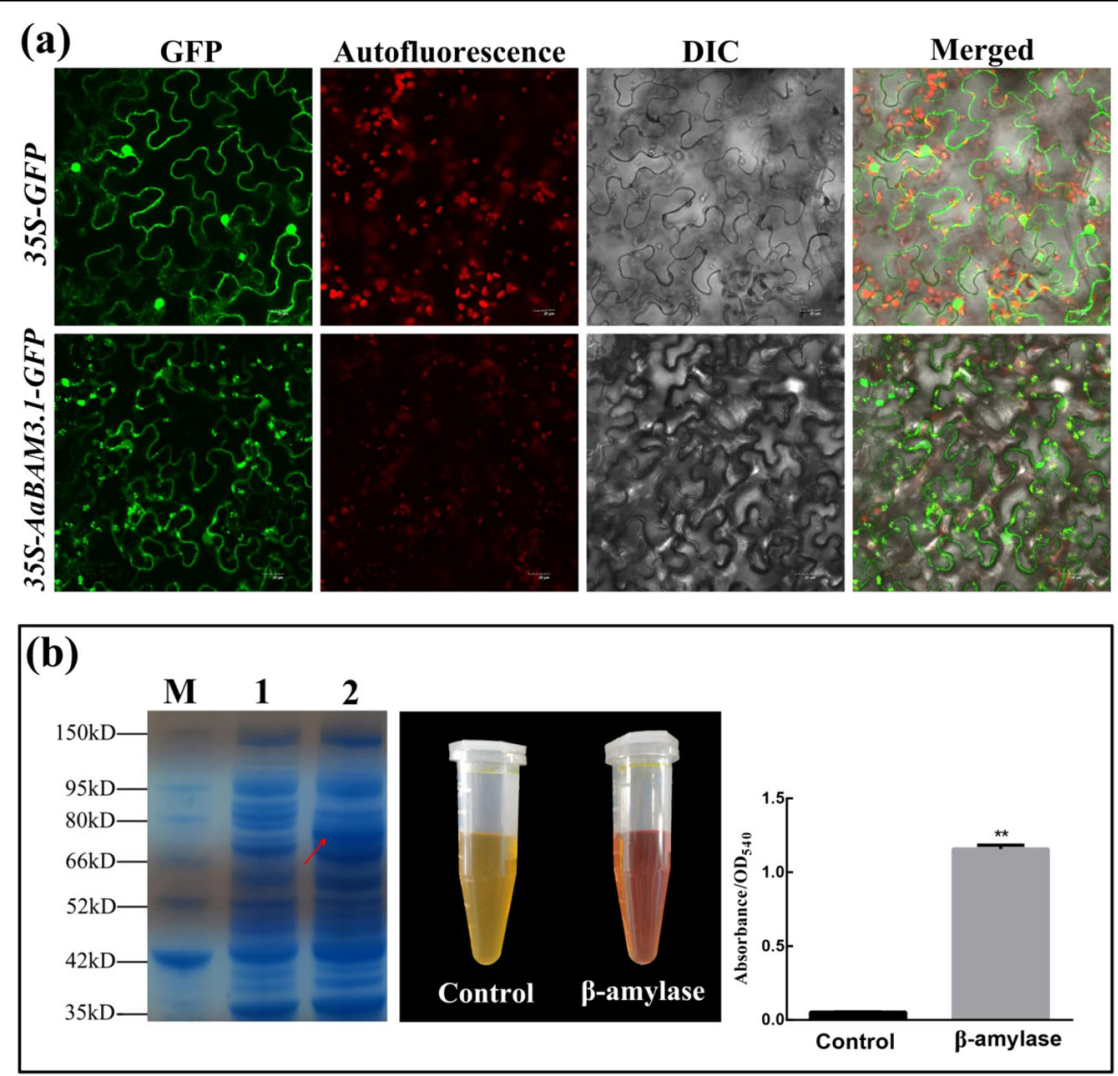

Fig. 2 Subcellular localization of AaBAM3.1-GFP and BAM activity in pGEX4T-1. a Tobacco leaves were injected with the control (35S-GFP) or recombinant plasmid (35S-AaBAM3.1-GFP) and visualized under a confocal microscope. GFP imaging and autofluorescence are shown. b SDS-PAGE analysis of the expression of the recombinant protein in $E$. coli. The total proteins from bacteria and the purified recombinant protein from the soluble crude extract were separated via 10\% SDS-PAGE and stained with Coomassie brilliant blue. M, protein size marker (15-150 kDa). Lane 1 contains proteins from uninduced cells, and lane 2 contains proteins from induced cells. The OD540 results obtained using a BAM enzyme kit showed that the recombinant protein presented enzymatic activity

A CBF binding site was identified in the promoter region of AaBAM3.1. ABRE, MYB, and ERF elements were also found in the promoter of $A a B A M 3.1$ (Table 1).

\section{Promoter activity assays for AaBAM3.1}

For beta-glucuronidase (GUS) histological staining assays, the $A a B A M 3.1$ promoter sequence was ligated into a pCAMBIA3301 vector to obtain the recombinant plasmid AaBAM3.1pro:GUS (Fig. 5a). The AaBAM3.1pro: GUS gene in the transgenic plants was induced and overexpressed during cold stress. The relative expression of GUS showed that its activity gradually increased during cold stress treatment (Fig. 5b). Taken together, the results showed that the AaBAM3.1 promoter played a key role in the response to cold stress.

\section{AaCBF expression during cold treatment and transcriptional activity assays}

Four $A a C B F$ genes were cloned and ligated into $A$. arguta using the homology-based cloning method; in accordance with the protocol for sequentially naming genes as they appear in the literature ${ }^{44}$, we named the four $A a C B F$ genes $A a C B F 1.1, A a C B F 2.1, A a C B F 2.2$, and AaCBF4 (GenBank Accession Nos. MT477463, MT477464, MT477465, and MT477466). The trend of the mRNA transcript levels of $A a C B F 2.2$ and AaCBF4 was the same as the expression of $A a B A M 3.1$ under cold treatment. In contrast, the expression of $A a C B F 1.1$ initially increased but then decreased with increasing treatment duration, and the $A a C B F 2.1$ transcript level remained low under cold treatment (Fig. 6a). Yeast strain AH109 containing pGBKT7-AaCBF2.2 and pGBKT7AaCBF4 grew well on SD/-Trp/-Ade/-His plates, whereas only yeast cells transformed with pGBKT7AaCBF4 exhibited GAL4 activity on SD/-Trp/-Ade/-His plates supplemented with $\mathrm{X}-\alpha-\mathrm{Gal}$, indicating that AaCBF4 presented transcriptional activation activity (Fig. 6b). The results of our subcellular localization analysis are shown in Fig. 6c. The AaCBF4::GFP fusion protein was localized in the nuclear region of tobacco 


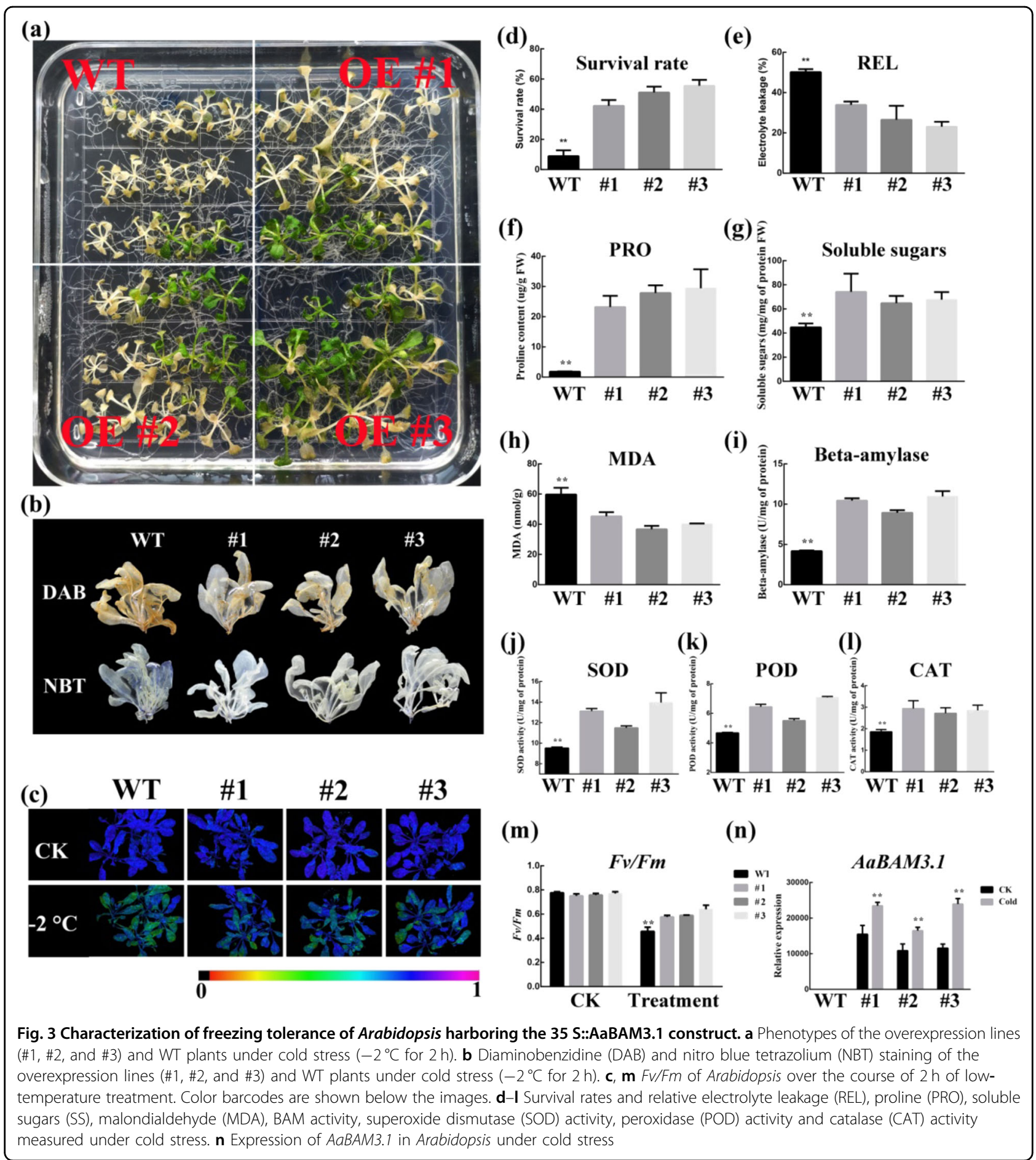

epidermal cells. Taken together, these results revealed that $A a C B F 4$ expression responded to cold exhibited and that this gene presented transcriptional activation activity.

\section{AaCBF4 binds to the AaBAM3.1 promoter}

Because the mRNA expression levels of AaBAM3.1 and $A a C B F 4$ increased in response to low temperature and because these genes play a key role in freezing tolerance, a cis-acting regulatory motif search of the AaBAM3.1 promoter was performed to determine whether AaCBF4 could regulate the expression of AaBAM3.1 under low temperature. We found a CBFbinding motif (TCGAC) located at $-1166 \mathrm{bp}$ in the AaBAM3.1 promoter (Fig. 7a). 

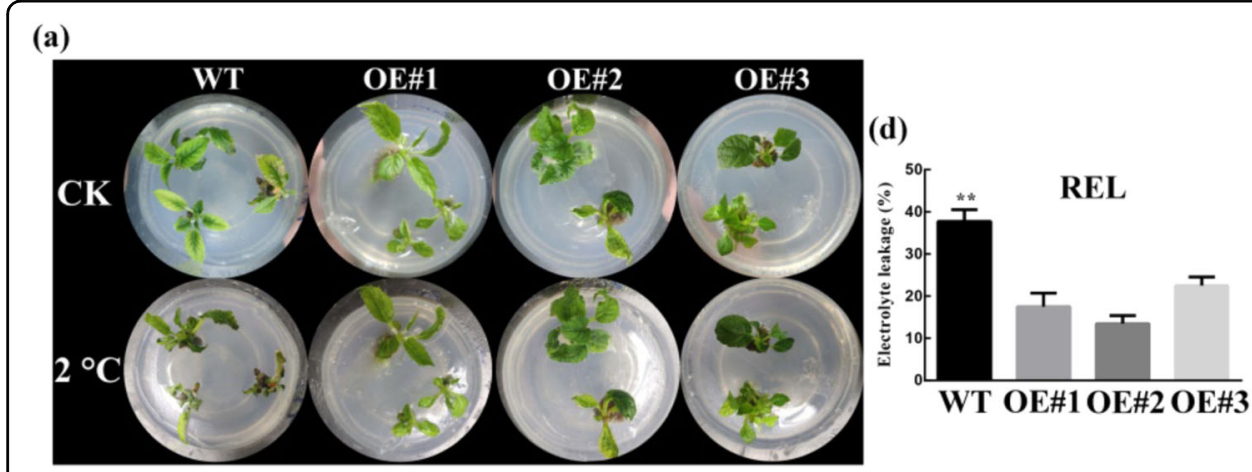

(e)

(b)
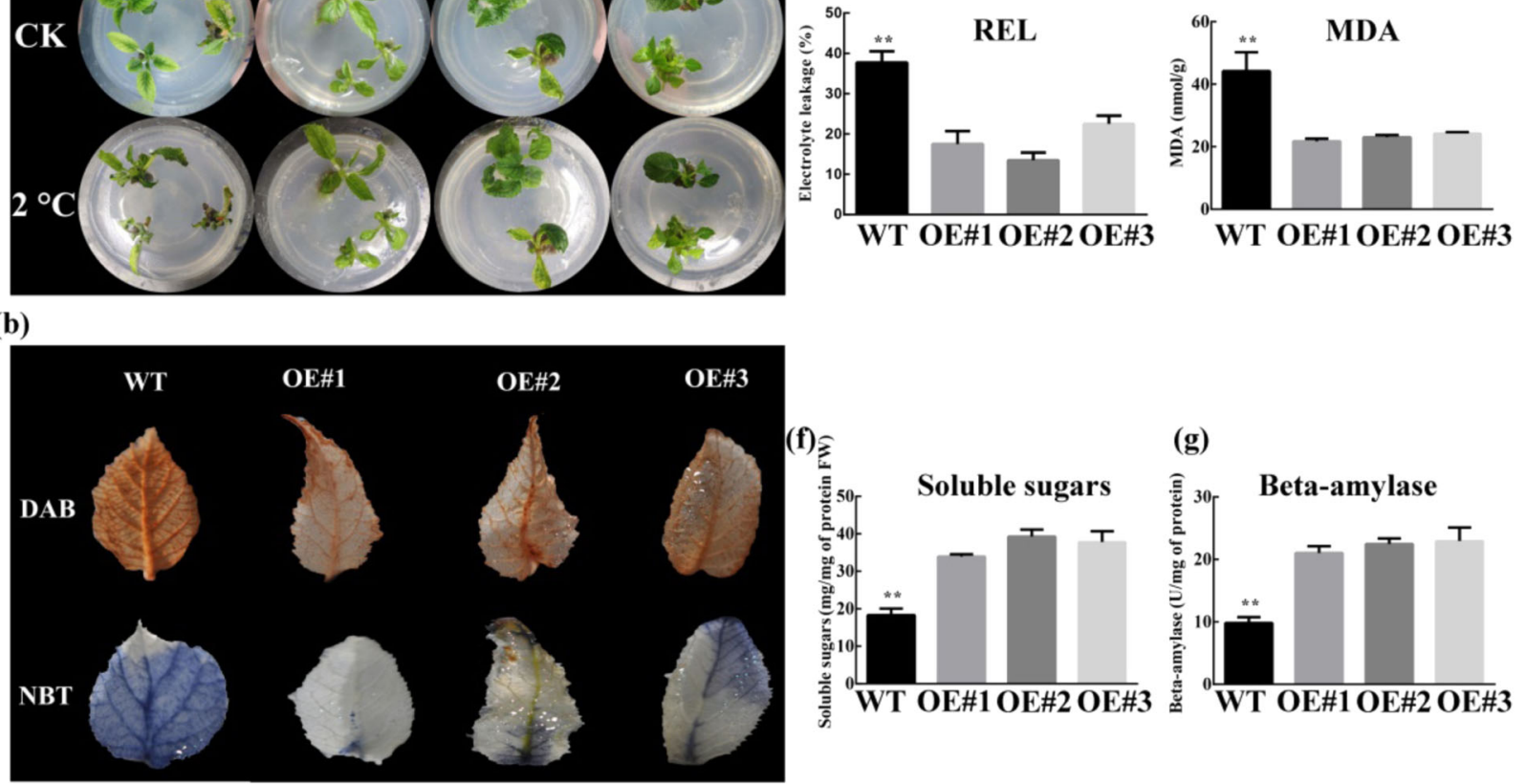

(c)

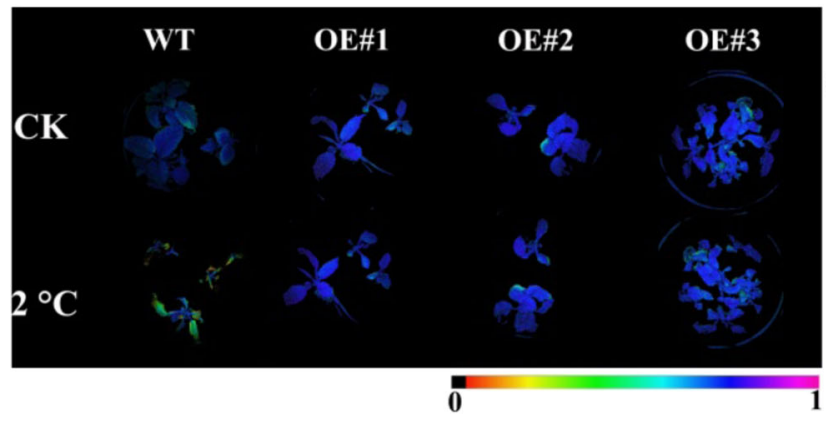

(h)

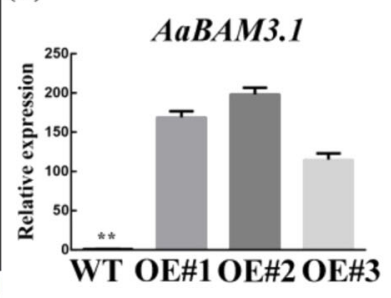

(g)

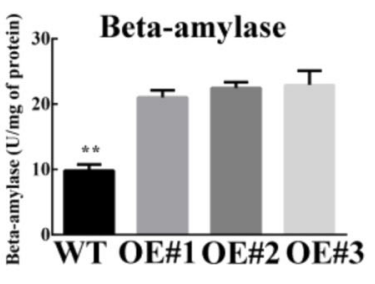

(i)

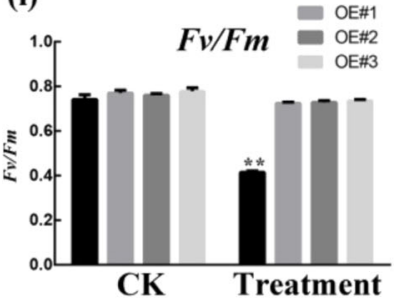

Fig. 4 Cold tolerance characterization of $A$. chinensis harboring the 35 S::AaBAM3.1 construct. a Phenotypes of the overexpression lines (\#1, $\# 2$, and \#3) and WT plants under cold stress $\left(2^{\circ} \mathrm{C}\right.$ for $2 \mathrm{~h}$ ). $\mathbf{b}$ Diaminobenzidine (DAB) and nitro blue tetrazolium (NBT) staining of the overexpression lines (\#1, \#2, and \#3) and WT plants under cold stress $\left(2^{\circ} \mathrm{C}\right.$ for $2 \mathrm{~h}$ ). $\mathbf{c}, \mathbf{i} \mathrm{FV} / \mathrm{Fm}$ of kiwifruit over the course of a $2 \mathrm{~h}$ low-temperature treatment. A colored barcode is shown below the images. $\mathbf{d}-\mathbf{g}$ Relative electrolyte leakage (REL), malondialdehyde (MDA), soluble sugars (SS) and BAM activity levels were measured under cold stress. (h) Expression of AaBAM3 in kiwifruit under cold stress

To verify the assumption that AaBAM3.1 expression is directly regulated by AaCBF4, we performed a yeast onehybrid assay (Y1H). The ORF of $A a C B F 4$ was cloned into the GAL4 activation domain in the pGADT7-Rec2 vector to construct the recombinant pGADT7-AaCBF4 plasmid (Fig. 7b). A $140 \mathrm{bp}$ region of the AaBAM3.1 promoter containing the CRT/DRE cis-element (TCGAC) was ligated and then cloned into a pAbAi vector to produce $\mathrm{p} P_{B A M 3.1}$-AbAi bait (Fig. 7b). The pGADT7-AaCBF4 prey vector and $\mathrm{p} P_{B A M 3.1}$-AbAi bait vectors were subsequently cotransformed into $\mathrm{Y} 1 \mathrm{H}$ strains, and the transformed $\mathrm{Y} 1 \mathrm{H}$ strains were then spread onto SD/-Leu media with or without $250 \mathrm{ng} / \mathrm{mL}$ AbA supplementation. pGADT7-p53 and p53-AbAi were used as positive controls. The pGADT7-AaCBF4 transformants and $\mathrm{p} P_{B A M 3.1}$-AbAi transformants grew well on media supplemented with $250 \mathrm{ng} / \mathrm{mL} \mathrm{AbA}$, and the positive control (harboring pGADT7-p53 and p53-AbAi) also grew well on media supplemented with $250 \mathrm{ng} / \mathrm{mL} \mathrm{AbA}$, whereas the negative control (harboring pGADT7-AaCBF4 and empty p-AbAi) could not grow in the presence of $250 \mathrm{ng} / \mathrm{mL} A b A$ (Fig. 7b). These results indicated that AaCBF4 bound to the CRT/DRE cis-element in the promoter of AaBAM3.1.

To validate the results of the $\mathrm{Y} 1 \mathrm{H}$ assays, we carried out a transient expression assay in tobacco leaves using a GUS activity assay. A $35 \mathrm{~S}:: \mathrm{AaCBF} 4$ fusion plasmid was 
constructed as an effector, and a recombinant $\mathrm{P}_{\mathrm{BAM} 3.1}:$ : GUS plasmid harboring $140 \mathrm{bp}$ of the AaBAM3.1 promoter sequence containing the CRT/DRE cis-element was used as a reporter. We observed that leaves cotransformed with $35 \mathrm{~S}:: \mathrm{AaCBF} 4$ and $\mathrm{P}_{\mathrm{BAM} 3.1}:$ GUS exhibited a deeper blue color than did the leaves injected with the empty pBI121 effector and $\mathrm{P}_{\mathrm{BAM} 3.1}:$ GUS; moreover, the relative GUS expression results were consistent with the GUS staining images (Fig. 7c).

Table 1 Cis-acting regulatory elements were predicted in the promoter regions of $A a B A M 3.1$ in relation to the cold response of $A$. arguta

\begin{tabular}{lll}
\hline $\begin{array}{l}\text { Cis-acting } \\
\text { element name }\end{array}$ & Sequence & Probable function \\
\hline CRT/DRE & TCGAC & $\begin{array}{l}\text { CBF (C-repeat-binding } \\
\text { factor)-binding }\end{array}$ \\
ABRE & ACGTC & Abscisic acid-responsiveness \\
MYB & CAACTG & \\
ERF & ATTT/CAAA & \\
TGACG-motif & TGACG & MeJA-responsiveness \\
Box4 & ATTAAT & Light responsiveness \\
MBS & CAACTG & Drought-inducibility \\
TCA-element & TCAGAAGAGG & Salicylic acid responsiveness \\
Circadian & CAAAGATATC & Circadian rhythm control \\
\hline
\end{tabular}

We further performed a dual-luciferase reporter assay to confirm the $\mathrm{Y} 1 \mathrm{H}$ results. The recombinant $35 \mathrm{~S}:$ : AaCBF4 effector and two reporters, $\mathrm{P}_{\mathrm{BAM} 3.1}:$ :LUC and pGreenII::LUC, containing $140 \mathrm{bp}$ AaBAM3.1 reporter sequences with or without the CRT/DRE cis-element, were constructed (Fig. $7 \mathrm{~d}$ ). The assays indicated that the LUC activities in the leaves infiltrated with $35 \mathrm{~S}:: \mathrm{AaCBF} 4$ and $P_{B A M 3.1}::$ LUC were significantly higher than those in the leaves injected with $35 \mathrm{~S}:: \mathrm{AaCBF} 4$ and pGreenII::LUC or with the pBI121 empty vector and $P_{B A M 3.1}:$ LUC (Fig. 7d). The results of the LUC activity imaging were consistent with the LUC activity values.

\section{Discussion}

\section{AaBAM3.1 is expressed in response to cold}

To survive cold stress, plants have developed the ability to resist low temperatures ${ }^{45}$. Many studies have revealed that plants degrade starch to produce soluble sugars and secondary metabolites to alleviate low-temperature injury ${ }^{46}$. Beta-amylase (mainly BAM3) is a major hydrolytic enzyme that attacks the nonreducing end of starch to release soluble sugars ${ }^{47}$. Among the nine $B A M$ genes present in A. thaliana, only AtBAM3 is transcriptionally induced by cold stress, which means that $A t B A M 3$ is a core gene involved in the mitigation of low-temperature injury in A. thaliana ${ }^{48}$. A previous study identified $16 A c B A M$ genes in the A. chinensis genome, and AaBAM3.1, obtained by homologous cloning, is a candidate gene for freezing tolerance in the dormant stage. According to the results of this study, AaBAM3.1 can be categorized together with the cold-responsive $B A M 3$ genes of other species (A. thaliana,

(a)

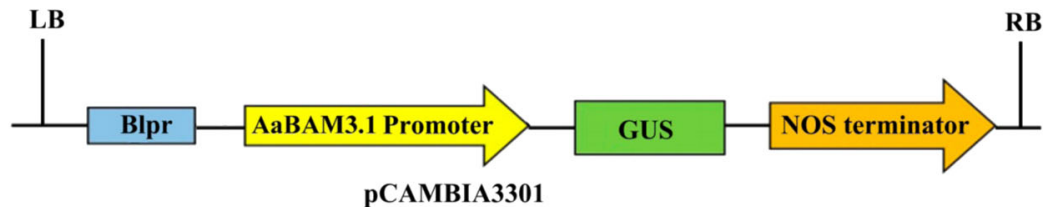

(b)

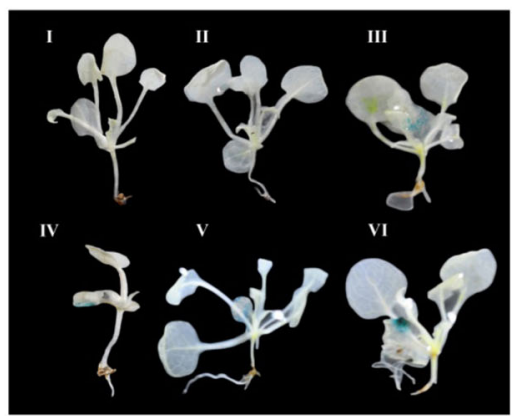

(c)

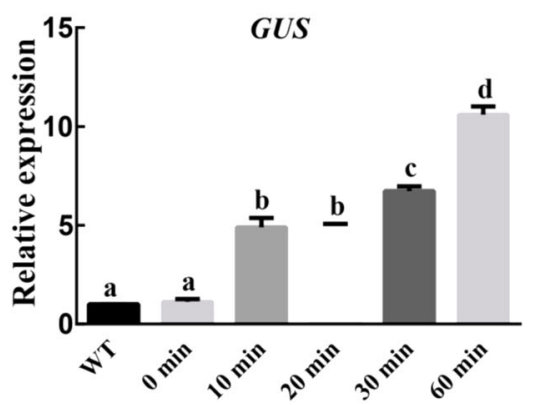

Fig. 5 Analysis of AaBAM3.1 promoter activity in transgenic Arabidopsis under cold stress. a Schematic diagram of PpCAMBIA3301-P $\mathrm{BAM}: \mathrm{G}$ GS. b Analysis of GUS activity in transgenic Arabidopsis expressing the AaBAM3.1 promoter under cold stress $\left(4^{\circ} \mathrm{C}\right)$. I: WT; II: transgenic plant ( ${ }^{\circ} \mathrm{C}$ for $0 \mathrm{~min})$; III: transgenic plant $\left(4^{\circ} \mathrm{C}\right.$ for $\left.10 \mathrm{~min}\right)$; IV: transgenic plant $\left(4^{\circ} \mathrm{C}\right.$ for $\left.20 \mathrm{~min}\right) ; \mathrm{V}$ : transgenic plant $\left(4^{\circ} \mathrm{C}\right.$ for $\left.30 \mathrm{~min}\right)$; Vl: transgenic plant $\left(4^{\circ} \mathrm{C}\right.$ for $60 \mathrm{~min})$. c GUS mRNA expression was measured via RT-qPCR at $4^{\circ} \mathrm{C}$ for $0 \mathrm{~min}, 10 \mathrm{~min}, 20 \mathrm{~min}, 30 \mathrm{~min}$, and $60 \mathrm{~min}$ 


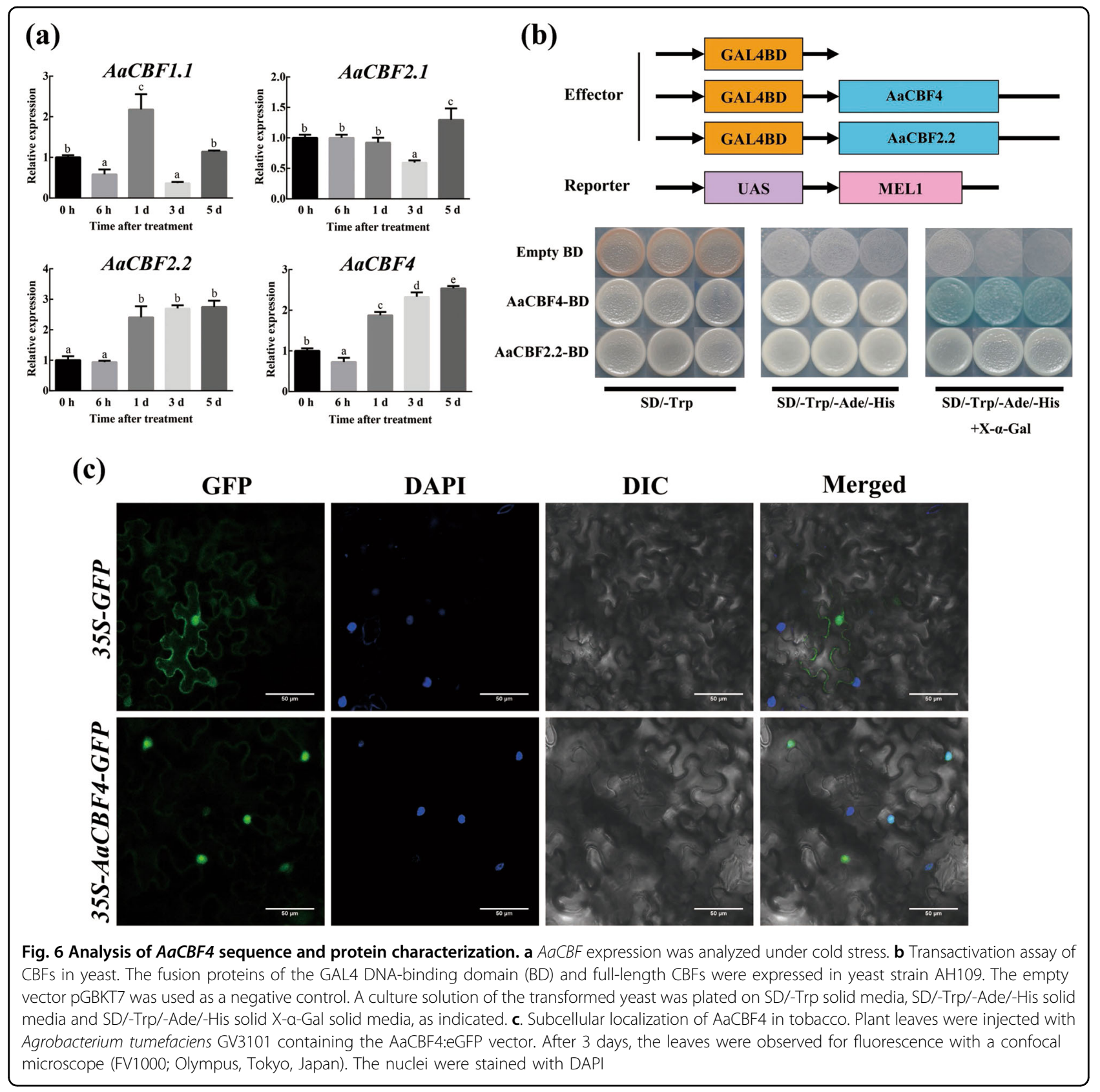

blueberry, orange, pear, tea) based on their sequence similarities and gene structure. In addition, BAM3 contains common domains that are conserved among different species. Based on these results, we can assume that starch metabolism is similar among plants in response to cold stress.

The activation of some BAM isoforms in response to cold stress has been reported according to gene expression and enzyme activity analyses. In rice, a $B M Y 8$ mutant was found to be cold sensitive due to an inability to break down $\operatorname{starch}^{33}$. In our study, the photosynthesis system and membrane integrity were hindered by cold treatment, which upregulated $A a B A M 3.1$ mRNA transcript levels. We can assume that $A a B A M 3.1$ is as a prominent $B A M$ gene involved in cold tolerance. First, AaBAM3.1 and AtBAM3 show high similarity in terms of domain conservation. Second, AaBAM3.1 mRNA transcript levels continuously increased in response to low temperature over 5 days of treatment. In the growth period, the evaluation of the tissue-specific expression of this gene showed that the mRNA transcript level was highest in the leaves and lowest in the shoots. In the dormant period, the expression levels increased rapidly in the shoots. Taken together, these results showed that the plants adopted 


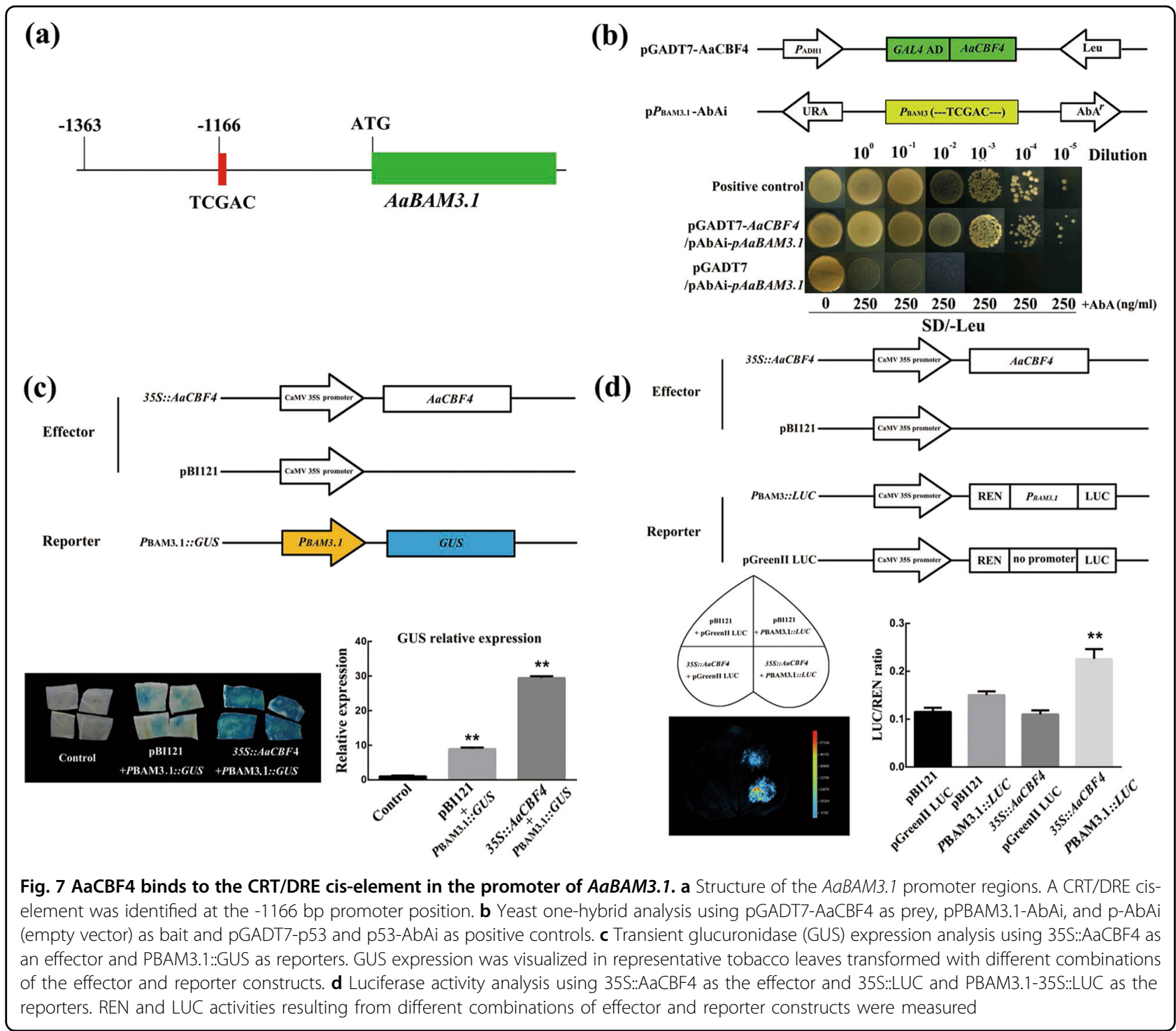

different strategies to cope with cold stress at different developmental stages in their annual growth cycle. Despite the different freezing tolerance strategies of the plants, AaBAM3.1 expression was continuously induced under low temperature, which meant that AaBAM3.1 played a role in the response to low-temperature stress and the enhancement of freezing tolerance at both the growth stage and the dormant stage.

\section{A. arguta AaBAM3.1 may play a role in enhancing freezing tolerance}

Cold stress is widely known to induce osmotic stress, which can trigger a decrease in membrane ion exchange, metabolic disorders, a loss of water potential and cell death, depending on the duration and degree of lowtemperature treatment ${ }^{49}$. Plants have developed sophisticated strategies for adapting to or surviving under cold stress, and one of these survival strategies involves the accumulation of very high levels of osmolytes or compatible solutes through osmotic adjustment ${ }^{50}$. Sugars are considered important osmolytes that can help plant cells resist abiotic stresses, improve water retention, regulate and stabilize biochemical reactions and protect membranes by adjusting the osmotic potential within the cell ${ }^{51}$. Sugar metabolism involves many pathways that produce large quantities of soluble sugars, such as sucrose, glucose, fructose, trehalose, fructans, and raffinose ${ }^{52}$. Plants treated with exogenous soluble sugars before cold stress have been shown to exhibit high accumulations of endogenous soluble sugars, showing that these metabolites and their derived products can act as compatible solutes that play key roles in protecting plant cells from injury induced by cold stress ${ }^{53}$. A positive correlation between the mRNA expression level of BAM3 and soluble sugar accumulation 
has been observed under cold stress, which leads to enhanced freezing tolerance ${ }^{54}$. Compared with WT plants, AtBAM3 RNAi lines show decreased soluble sugar levels and poor freezing tolerance under cold stress, indicating that these characteristics might be positively correlated. In this study, the higher accumulation of compatible solutes observed in AaBAM3.1-overexpressing transgenic lines (both $A$. thaliana and kiwifruit plants) led us to speculate that the transgenic plants overexpressing AaBAM3.1 may have a stronger osmotic adjustment ability than WT plants have. This assumption was supported by the low level of REL observed under low temperature, indicating that, compared with the WT plants, the AaBAM3.1-overexpressing lines experienced minor osmotic stress injury. Consequently, the higher accumulation of soluble sugars was demonstrated to be one of the survival mechanisms enhancing freezing tolerance in the AaBAM3.1-overexpressing transgenic lines compared with the WT plants.

Cold stress disrupts the redox balance in cells, which leads to the accumulation of additional $\operatorname{ROS}^{55}$. In turn, high ROS accumulation leads to oxidative stress, which is harmful to cellular functions and biological processes because of the denaturation of functional proteins ${ }^{56}$. Under nonstress conditions, the amount of ROS production is equal to that of ROS scavenging, while increased ROS accumulation is mainly due to an imbalance between ROS production and scavenging under cold stress. To survive oxidative stress, plants have evolved complex strategies to scavenge various types of ROS. Some of the well-known antioxidant compounds can be divided into two categories: enzymatic (SOD, POD, CAT, etc.) and nonenzymatic (anthocyanin, carotenoids, glutathione, tocopherols and ascorbic acid, etc.) ones ${ }^{57-60}$. Recent findings have shown that several sugars can serve as antioxidants and have an important effect on ROS scavenging, including glucose, fructose, sucrose, inositol, galactinol, trehalose and raffinose $^{18}$. It is clear that the regulation of the plant redox system can increase freezing tolerance ${ }^{61}$. In this study, DAB and NBT staining assays indicated that the accumulation of antioxidants in AaBAM3.1-overexpressing plants was greater than that in WT plants, suggesting that the AaBAM3.1-overexpressing plants present a robust ability to scavenge ROS under cold stress. Moreover, the lower accumulation of MDA, which serves as an indicator of the degree of membrane damage, showed that the AaBAM3.1overexpressing transgenic lines experienced minor oxidative stress. Chloroplasts and mitochondria are the major organelles responsible for ROS generation due to the disorder of the electron transfer chain. Interestingly, subcellular localization results showed that AaBAM3.1 localizes to the chloroplast, implying that the AaBAM3.1 enzyme in chloroplasts might be directly and indirectly involved in scavenging ROS. Soluble sugars derived from starch degradation in chloroplasts can play a key role in sugar metabolism to produce various sugar derivatives through a set of sugar metabolism pathways. Therefore, we assume that, compared with the WT plants, the overexpressing lines accumulated greater amounts of soluble sugars and their derivatives to scavenge the ROS produced in the chloroplasts. ROS produced in the cytoplasm can also be scavenged by soluble sugars ${ }^{62}$. Overall, the results revealed that the increased ROS-scavenging ability derived from the highly abundant accumulation of soluble sugars is a survival mechanism that enhances the freezing tolerance of kiwifruit.

\section{A model for the regulation of freezing tolerance driven by the AaCBF4-AaBAM3.1 module}

The $B A M$ gene is a functional gene encoding an enzyme with catalytic activity. Moreover, it plays a direct role in alleviating cold damage by producing soluble sugars contributing to cold stress resistance. However, the specific role of the $B A M$ gene in transcriptional regulatory networks is poorly understood. The OsMYB30 transcription factor can suppress the expression of $B M Y 2$, $B M Y 6$, and $B M Y 10$ in rice ${ }^{63}$. In the past few decades, the $\mathrm{CBF}$ regulatory pathway has become the best-understood cold-responsive network ${ }^{64}$. It has been shown that CBFs act as a hub linking the downstream low-temperature response with upstream signal transmission. In some species, including $A$. thaliana, Triticum aestivum, Hordeum vulgare, and Brassica oleracea, some $C B F$ genes have been shown to serve as indicators of freezing tolerance. The expression of a number of COR genes is regulated by CBFs, which bind to corresponding CRT/ DRE cis-elements in the promoters of the COR genes. The genes containing CRT/DRE cis-elements in their promoters encode a series of different cryoprotectant proteins, including COR15a, ERD10, COR6.6, KIN1, RD19a, and COR47, which play key roles in enhancing freezing tolerance ${ }^{65}$. However, although some COR genes containing CRT/DRE cis-elements in their promoters have been identified, the knowledge of the genes regulated by CBFs and their functions in freezing tolerance of kiwifruit is still quite poor. In this study, we found that the $A a C B F 4$ gene could respond to cold stress and tended to be expressed in the same manner as the AaBAM3.1 gene was under cold stress. The AaCBF4 gene encodes a TF involved in the positive regulation of the freezing tolerance of kiwifruit. A CBF-binding element has been identified in the AaBAM3.1 promoter region. $\mathrm{Y} 1 \mathrm{H}$, transient GUS and dual-luciferase reporter assays indicated that AaCBF4 showed a positive relationship with AaBAM3.1. On the basis of these results, we speculated that $A a B A M 3.1$ expression is regulated by $\mathrm{AaCBF} 4$, indicating that the $A a C B F-A a B A M$ module may be activated by cold stress to enhance freezing tolerance. 


\section{Conclusion}

In conclusion, our results demonstrate that AaBAM3.1, which is localized in the chloroplasts, is responsible for freezing tolerance enhancement and is involved in starch degradation. AaBAM3.1 plays a key role in enhancing freezing tolerance through the modulation of compatible solute and antioxidant enzyme levels. AaBAM3.1 is regarded as a cold-responsive gene containing CRT/DRE elements in its promoters. Hence, these findings show that the AaCBF4-AaBAM3.1 module is involved in the transduction network related to the freezing tolerance of kiwifruit.

\section{Materials and methods}

\section{Plant materials and treatments}

Two Actinidia species, A. arguta cv. Kuilv (KL) (whose semilethal temperature is $-30^{\circ} \mathrm{C}$ ) and $A$. chinensis cv. Hongyang (whose semilethal temperature is $-13^{\circ} \mathrm{C}$ ) were used in this study. Micropropagated plantlets were grown on Murashige and Skoog 1962 (MS) culture media comprising $7.2 \mathrm{~g} / \mathrm{L}$ agar and $30 \mathrm{~g} / \mathrm{L}$ sucrose $(\mathrm{pH}$ 5.8). Thirty-day-old plants displaying uniform growth were used for cold treatments. Plants grown in bottles were treated at $4{ }^{\circ} \mathrm{C}$ in a low-temperature incubator, and whole leaves were then harvested at $0 \mathrm{~h}, 6 \mathrm{~h}, 1 \mathrm{~d}, 3 \mathrm{~d}$, and $5 \mathrm{~d}$ time points. Each sample included three biological replicates. For seasonal gene expression samples, mature (3-year-old) KL plants planted in 3-1 pots were placed in the field. The shoots were collected from mid-April 2017 to March 2018. All six of the collection points were in the morning: S1, 15 April; S2, 15 November; S3, 7 December; S4, 23 December; S5, 15 January; and S6, 15 March. Moreover, different tissues of KL plants were collected. Three biological replicates of shoots were used for RNA extraction.

\section{Gene cloning and sequence analysis}

The open reading frames (ORFs) of AaBAM3.1 and AaCBF4 were cloned from the cDNA of $A$. arguta (KL) leaves by using the primers AaBAM3.1-ORF-F/R and AaCBF4-ORF-F/R (Table S1). AaCBF1.1, AaCBF2.1 and AaCBF2.2 were cloned by using the primers AaCBF1.1ORF-F/R, AaCBF2.1-ORF-F/R and AaCBF2.2-ORF-F/R, respectively. Multiple alignment of the putative protein sequences was conducted via DNAMAN 7.0. MEGAX 32 software was utilized to construct a maximum likelihood (ML) tree with 1000 bootstrap replicates. Tools from the online website ExPASy (http://www.expasy.org) were used to predict the molecular weights and theoretical isoelectric points. Furthermore, the ClustalX program with default settings was employed to predict the conserved domains. Underlying cis-elements in the promoter sequence were identified via the NewPLACE (https://www.dna.affrc.go.jp/PLACE/) database.

\section{Quantitative real-time PCR (RT-qPCR) analysis}

The RNA extraction method and RT-qPCR were performed as described by $\mathrm{Li}^{66}$. Three independent biological replicates were analyzed for each sample. All the data were analyzed using the $2^{-\triangle \triangle C t}$ method $^{67}$. The RTqPCR primers were designed by using Primer Premier 5 (the primers used are listed in Table S1).

\section{Subcellular localization of AaBAM3.1 and AaCBF4}

The coding sequences of $A a B A M 3.1$ and $A a C B F 4$ were amplified via $\mathrm{HiFi}$-enzyme PCR using primers without stop codons. The PCR products of AaBAM3.1 and $A a C B F 4$ were then ligated into a pBI121 plasmid and fused together with GFP, and their expression was driven by the CaMV $35 \mathrm{~S}$ promoter. Following sequence confirmation, the recombinant $35 \mathrm{~S}$ pro:AaBAM3.1::GFP, $35 \mathrm{~S}$ pro:AaCBF4::GFP, and $35 \mathrm{~S}$ pro:GFP (control) plasmids were transferred into Agrobacterium tumefaciens strain GV3101. Tobacco leaves were injected with these bacterial suspensions $\left(\mathrm{OD}_{600}=0.6-1.0\right)$, which were then kept in a chamber for 2-3 days, after which the transformed leaves were then observed under a confocal laserscanning microscope (FV1000; Olympus, Tokyo, Japan).

\section{Detection of BAM enzyme activity}

The AaBAM3.1 cDNA was cloned by using primers without stop codons. The PCR product was subcloned into pGEX4T-1 with a GST tag. The fusion plasmid and EV were subsequently transferred into the $E$. coli Rosetta (DE3) strain. E. coli cells harboring pGEX4T-1-AaBAM3.1 and pGEX4T-1 (EV) were then cultivated in $50 \mathrm{~mL}$ of liquid LB media consisting of $50 \mathrm{mg} / \mathrm{L}$ kanamycin at $37^{\circ} \mathrm{C}$ to an $\mathrm{OD}_{600}=0.6$ and then induced with $1 \mathrm{mM}$ isopropyl-betaD-thiogalactopyranoside (IPTG) at $28^{\circ} \mathrm{C}$. After $8 \mathrm{~h}$, the cells were collected by centrifugation at $10,000 \times \mathrm{g}$ for $10 \mathrm{~min}$, resuspended in phosphate-buffered saline (PBS; $\mathrm{pH}=7.0$ ), and disrupted by sonication. Enzyme activity was measured using a beta-amylase assay kit (Nanjing Jiancheng Bioengineering Institute, China).

\section{$\mathrm{Y} 1 \mathrm{H}$ assays}

$\mathrm{Y} 1 \mathrm{H}$ assays were conducted using a Matchmaker ${ }^{\mathrm{TM}}$ Gold Yeast One-Hybrid Library Screening System (Clontech, San Francisco, USA) to examine the interaction of AaCBF4 and the AaBAM3.1 promoter. The primers listed in Table S1 were used to clone the $140 \mathrm{bp}$ promoter region containing a CRT/DRE cis-element. The amplified $140 \mathrm{bp}$ sequence was cloned into an pAbAi vector to construct bait. The AaCBF4 ORF was fused with the GAL4 activation domain (AD) in the pGADT7$A D$ vector to produce a prey vector (pGAD-AaCBF4). After the transformants were screened on SD/-Ura plates and the minimal inhibitory concentration of aureobasidin A (AbA) for the positive bait strains was measured, 
pGAD-AaCBF4 was transferred to yeast cells. A positive yeast strain was selected on SD/-Leu plates that included $250 \mathrm{ng} / \mathrm{mL} \mathrm{AbA}$ and were cultured at $30^{\circ} \mathrm{C}$ for $2-3$ days. Positive yeast cells harboring pGADT7-53 and p53-AbAi were used as positive controls.

\section{GUS and luciferase reporter assays}

To verify the interaction between AaCBF4 and the CRT/ DRE cis-elements in the AaBAM3.1 promoter, transient expression experiments and GUS and luciferase reporter assays were conducted in the model plant species tobacco $^{68}$. The PCR-amplified $140 \mathrm{bp}$ promoter sequence containing the AaBAM3.1 promoter CRT/DRE cis-element was cloned into pBI121 and pGreenII 0800-LUC vectors to obtain reporter plasmids. The AaCBF4 ORF was ligated into pBI121 to produce effector constructs. The effector and reporter constructs were subsequently transferred into A. tumefaciens strain GV3101, which were then coinfiltrated into tobacco leaves via agroinfiltration ${ }^{69}$. After coculturing in an illuminated chamber for $48-72 \mathrm{~h}$ at $25^{\circ} \mathrm{C}$, a Dual-Luciferase Reporter Assay System (Promega, USA) was used to determine LUC and REN luciferase activities according to the manufacturer's instructions, and GUS staining was performed as described by Hellens ${ }^{70}$.

\section{A. thaliana transformation}

The AaBAM3.1 ORF driven by the constitutive CaMV $35 \mathrm{~S}$ promoter or $A a B A M 3.1$ promoter was cloned into pBI121 or pCAMBIA3301. A. tumefaciens (strain GV3101) harboring the AaBAM3.1 ORF or AaBAM3.1 promoter-containing recombinant plasmids were transformed into WT A. thaliana (Col-0) plants via the floraldip method $^{71}$. T0 seeds were selected on MS screening culture media comprising kanamycin (Kan) $(100 \mathrm{mg} / \mathrm{L}$; Sigma, St. Louis, USA) or phosphinothricin (Basta) $(1 \mathrm{mg} /$ L; Sigma, St. Louis, USA). Three positive T3 transgenic lines were chosen for further gene functional verification.

\section{Cold treatment of transgenic $A$. thaliana and analysis of physiological characteristics}

For the freezing tolerance experiment, 3-week-old WT and transgenic $A$. thaliana plants (Fig. S1) were treated as described by Ding ${ }^{72}$. The survival was scored 7 days after treatment. For the determination of physiological changes, 3 -week-old $A$. thaliana plants from the WT and transgenic lines were subjected to treatment at $-2{ }^{\circ} \mathrm{C}$ for $2 \mathrm{~h}$. Afterward, SOD, POD, CAT, and BAM activities and PRO, SS and MDA contents were determined as described in the kit manual (Nanjing Jiancheng Bioengineering Institute, China). REL was measured via the method described by Lappi ${ }^{73}$. Using the 3,3'-diaminobenzidine (DAB) and nitro blue tetrazolium (NBT) staining methods, the accumulation of hydrogen peroxide $\left(\mathrm{H}_{2} \mathrm{O}_{2}\right)$ and superoxide $\left(\mathrm{O}_{2}{ }^{-}\right)$under cold stress was determined ${ }^{74,75}$. For chlorophyll fluorescence measurements, whole plants were imaged using an IMAGING-PAM chlorophyll fluorometer (Walz, Effeltrich, Germany), and plants were placed in a dark room for $20 \mathrm{~min}$ prior to imaging. The maximum quantum efficiency of photosystem II $(F v / F m)$ was measured with Imaging WinGegE software ${ }^{76}$. For GUS staining, 3-week-old A. thaliana plants (Fig. S3) from the WT and transgenic lines were subjected to $4{ }^{\circ} \mathrm{C}$ for $0 \mathrm{~min}, 10 \mathrm{~min}, 20 \mathrm{~min}, 30 \mathrm{~min}$, or $60 \mathrm{~min}$. Each sample consisted of three seedlings, and each experiment was performed in triplicate.

\section{Transformation of $A$. chinensis leaves and regeneration of transgenic plants}

The AaBAM3.1 ORF was cloned into a pBI121 vector driven by the CaMV $35 \mathrm{~S}$ promoter, and the recombinant plasmid was subsequently transformed into A. chinensis leaves based on the protocol outlined by Wang ${ }^{77}$ (Fig. S2). Transgenic plants were obtained after $\sim 6$ months. The transformed plants were identified using PCR and RTqPCR methods to successfully verify the incorporation of the transgene. Three positive transgenic kiwifruit lines with high $A a B A M 3.1$ mRNA expression were chosen for molecular and phenotypic analyses.

\section{Evaluation of the cold tolerance of AaBAM3.1- overexpressing $A$. chinensis plants}

$A$. chinensis plants from the WT and three transgenic lines (OE\#1, OE\#2, OE\#3) were subjected to cold stress (at $2{ }^{\circ} \mathrm{C}$ ) for $2 \mathrm{~h}$. After cold treatment, plant leaves were harvested to measure various physiological indicators. The BAM activity and SS contents were determined based on the abovementioned methods. The REL and MDA contents were measured according the abovementioned methods. The in situ accumulation of $\mathrm{H}_{2} \mathrm{O}_{2}$ and $\mathrm{O}_{2}{ }^{-}$was detected via DAB and NBT, respectively. For chlorophyll fluorescence detection, whole plants were imaged by an IMAGING-PAM chlorophyll fluorometer, and the maximum quantum efficiency of photosystem II $(F v / F m)$ was determined by Imaging WinGegE software.

\section{Statistical analysis}

The statistical analysis was performed with the Windows-based SPSS software package (version 22, USA). T-tests and one-way ANOVA were used to analyze the data, and Duncan's multiple comparisons were employed for sample comparisons at significance levels of $P<0.05$ or $P<0.01$.

\section{Acknowledgements}

This study was funded by the National Science Foundation of China (Grant No. 31801820); the National Key Research and Development Project of China (Grant No. 2019YFD1000800); the Special Engineering Science and Technology Innovation, Chinese Academy of Agricultural Sciences (Grant No. CAAS-ASTIP2015-ZFRI); and the Modern Agricultural Industrial Technology System of Henan Province (Grant No. S2014-11). 


\section{Author contributions}

S.S., M.L., X.Q., J.C., Y.Z., and A.M. conducted the experiments; J.F. organized and supervised the overall project; S.S. and M.L. performed the data analysis and wrote the manuscript writing; and C.H. edited the manuscript.

\section{Conflict of interest}

The authors declare no competing interests.

Supplementary information The online version contains supplementary material available at https://doi.org/10.1038/s41438-021-00530-1.

Received: 10 November 2020 Revised: 20 January 2021 Accepted: 24 February 2021

Published online: 01 May 2021

\section{References}

1. Kou, S. et al. The arginine decarboxylase gene $A D C 1$, associated to the putrescine pathway, plays an important role in potato cold-acclimated freezing tolerance as revealed by transcriptome and metabolome analyses. Plant J. 96, 1283-1298 (2018).

2. Stockinger, E. J., Gilmour, S. J. \& Thomashow, M. F. Arabidopsis thaliana CBF1 encodes an AP2 domain-containing transcriptional activator that binds to the C-repeat/DRE, a cis-acting DNA regulatory element that stimulates transcription in response to low temperature and water deficit. Proc. Natl Acad. Sci. USA 94, 1035-1040 (1997)

3. Wisniewski, M., Bassett, C. \& Gusta, L. V. An overview of cold hardiness in Woody plants: Seeing the forest through the trees. Hortscience 38, 952-959 (2003).

4. Wisniewski, M., Nassuth, A. \& Arora, R. Cold hardiness in trees: a mini-review. Front. Plant Sci. 9, 1-9 1394 (2018).

5. Chen, M. J. et al. Characterization of low temperature-induced plasma membrane lipidome remodeling combined with gene expression analysis reveals mechanisms that regulate membrane lipid desaturation in Carica papaya. Sci. Hortic. 272, 1-12 (2020). 109505.

6. Ma, Y. et al. COLD1 confers chilling tolerance in rice. Cell 162, 222-222 (2015).

7. Zhao, C. Z. et al. MAP kinase cascades regulate the cold response by modulating ICE1 protein stability. Dev. Cell 43, 618-629 (2017).

8. He, Y. A. et al. Phytochrome B negatively affects cold tolerance by regulating OsDREB1 gene expression through phytochrome interacting factor-like protein OsPIL16 in rice. Front. Plant Sci. 7, 1-12 (2016).

9. $\mathrm{Li}, \mathrm{H}$. et al. BZR1 positively regulates freezing tolerance via CBF-dependent and CBF-independent pathways in arabidopsis. Mol. Plant. 10, 545-559 (2017).

10. $\mathrm{Hu}, \mathrm{H}$. H. \& Xiong, L. Z. Genetic engineering and breeding of drought-resistant crops. Annu. Rev. Plant Biol. 65, 715-741 (2014).

11. Dreyer, A. \& Dietz, K. J. Reactive oxygen species and the redox-regulatory network in cold stress acclimation. Antioxid.-basel 7, 1-15 (2018).

12. Chu, M. X. et al. AtCaM4 interacts with a Sec14-like protein, PATL1, to regulate freezing tolerance in Arabidopsis in a CBF-independent manner. J. Exp. Bot. 69 , 5241-5253 (2018).

13. Ishikawa, M. et al. Ice Nucleation Activity in Plants: The Distribution, Characterization, and Their Roles in Cold Hardiness Mechanisms in Survival Strategies in Extreme Cold and Desiccation: Adaptation Mechanisms and Their Applications Vol. 1081 (eds Iwayalnoue, M., Sakurai, M. \& Uemura, M.) 99-115 (Springer-Verlag Singapore Pte Ltd, 2018).

14. Karami, H., Rezaei, M., Sarkhosh, A., Rahemi, M. \& Jafari, M. Cold hardiness assessment in seven commercial fig cultivars (Ficus CaricaL). Gesund. Pflanz. 70, 195-203 (2018).

15. Zhang, H., Wang, Y. P., Wang, W. E., Bao, M. Z. \& Chan, Z. L. Physiological changes and DREB1s expression profiles of tall fescue in response to freezing stress. Sci. Hortic. 245, 116-124 (2019).

16. Miller, G., Suzuki, N., Ciftci-Yilmaz, S. \& Mittler, R. Reactive oxygen species homeostasis and signalling during drought and salinity stresses. Plant Cell Environ. 33, 453-467 (2010).

17. Cabello, J. V., Lodeyro, A. F. \& Zurbriggen, M. D. Novel perspectives for the engineering of abiotic stress tolerance in plants. Curr. Opin. Biotechnol. 26, 62-70 (2014)
18. Keunen, E., Peshev, D., Vangronsveld, J., Van den Ende, W. \& Cuypers, A. Plant sugars are crucial players in the oxidative challenge during abiotic stress: extending the traditional concept. Plant Cell Environ. 36, 1242-1255 (2013).

19. Dietz, K. J. Redox signal integration: from stimulus to networks and genes Physiol. Plant. 133, 459-468 (2008).

20. Adams, C. A. \& Rinne, R. W. The occurrence and significance of dispensable proteins in plants. N. Phytol. 89, 1-14 (1981).

21. Hildebrand, D. F. \& Hymowitz, T. The Sp1 locus in soybean codes for beta -amylase. Crop Sci. 20, 165-168 (1980).

22. Thalmann, M. \& Santelia, D. Starch as a determinant of plant fitness under abiotic stress. N. Phytol. 214, 943-951 (2017).

23. Sun, S. H. et al. Freezing tolerance and expression of beta-amylase gene in two actinidia arguta cultivars with seasonal changes. Plants-Basel 9, 1-12 515 (2020).

24. Fulton, D. C. et al. $\beta$-AMYLASE4, a noncatalytic protein required for starch breakdown, acts upstream of three active $\beta$-amylases in arabidopsis chloroplasts. Plant Cell 20, 1040-1058 (2008).

25. Zeeman, S. C. et al. Plastidial alpha-glucan phosphorylase is not required for starch degradation in Arabidopsis leaves but has a role in the tolerance of abiotic stress. Plant physiol. 135, 849-858 (2004).

26. Hyung, L. J., Jun, Y. D., Jin, K. S., Doil, C. \& Jae, L. H. Intraspecies differences in cold hardiness, carbohydrate content and $\beta$-amylase gene expression of Vaccinium corymbosum during cold acclimation and deacclimation. Tree Physiol. 32, 1533-1540 (2012).

27. Hao, X. Y., Yue, C., Tang, H., Qian, W. J. \& Yang, Y. J. Cloning of $\beta$-amylase Gene (CsBAM3) and Its Expression Model Response to Cold Stress in Tea Plant. Acta Agronomica Sin. 43, 1417-1425 (2017).

28. Peng, T., Zhu, X., Duan, N. \& Liu, J. H. PtrBAM1, a $\beta$-amylase-coding gene of Poncirus trifoliata, is a CBF regulon member with function in cold tolerance by modulating soluble sugar levels. Plant Cell Environ. 37, 2754-2767 (2015).

29. Liangyi et al. Transcriptomic and evolutionary analyses of white pear (Pyrus bretschneideri) $\beta$-amylase genes reveals their importance for cold and drought stress responses. Gene 689, 102-113 (2018).

30. Nielsen, T. H., Deiting, U. \& Stitt, M. A beta-amylase in potato tubers is induced by storage at low temperature. Plant physiol. 113, 503-510 (1997).

31. Dong, S. Y. \& Beckles, D. M. Dynamic changes in the starch-sugar interconversion within plant source and sink tissues promote a better abiotic stress response. J. Plant Physiol. 234, 80-93 (2019).

32. Monroe, J. D. \& Storm, A. R. Review: the arabidopsis $\beta$-amylase (BAM) gene family: diversity of form and function. Plant Sci. 276, 163-170 (2018).

33. Kaplan, F. \& Guy, C. L. RNA interference of Arabidopsis beta-amylase8 prevents maltose accumulation upon cold shock and increases sensitivity of PSII photochemical efficiency to freezing stress. Plant J. 44, 730-743 (2010).

34. Hou, J. et al. Amylases StAmy23, StBAM1 and StBAM9 regulate cold-induced sweetening of potato tubers in distinct ways. J. Exp. Bot. 68, 2317-2331 (2017).

35. An, J. P. et al. MdHY5 positively regulates cold tolerance via CBF-dependent and CBF-independent pathways in apple. J. Plant Physiol. 218, 275-281 (2017).

36. Lee et al. The unified ICE-CBF pathway provides a transcriptional feedback control of freezing tolerance during cold acclimation in Arabidopsis. Plant Mol. Biol. 89, 187-201 (2015).

37. Cook, D., Fowler, S., Fiehn, O. \& Thomashow, M. F. A prominent role for the CBF cold response pathway in configuring the low-temperature metabolome of Arabidopsis. Proc. Natl Acad. Sci. USA 101, 15243-15248 (2004).

38. Feng, W. Q., Li, J., Long, S. X. \& Wei, S. J. A DREB1 gene from zoysiagrass enhances Arabidopsis tolerance to temperature stresses without growth inhibition. Plant Sci. 278, 20-31 (2019).

39. Mizoi, J., Shinozaki, K. \& Yamaguchi-Shinozaki, K. AP2/ERF family transcription factors in plant abiotic stress responses. Biochim. Biophys. Acta-Gene Regul. Mech. 1819, 86-96 (2012).

40. Cho, S. et al. Accession-dependent CBF gene deletion by CRISPR/Cas system in Arabidopsis. Front. Plant Sci. 8, 1-11 1910 (2017).

41. Liu, Y. F. et al. Rapid radiations of both kiwifruit hybrid lineages and their parents shed light on a two-layer mode of species diversification. N. Phytol. 215, 877-890 (2017).

42. Sun, S., Qi, X., Wang, R., Lin, M. \& Fang, J. Evaluation of freezing tolerance in Actinidia germplasm based on relative electrolyte leakage. Hortic. Environ. Biote. 61, 755-765 (2020).

43. Jo, Y. S., Cho, H. S., Park, J. O., Kim, T. C. \& Kim, W. S. Assay of Cold Tolerance of Actinidia eriantha in Proceedings of the International Symposium on Citrus and Other Tropical and Subtropical Fruit Crops Vol. 41 (ed Oh, D. G.) 277-282 (Int Soc Horticultural Science, 2008). 
44. Gunaseelan, K. et al. Copy number variants in kiwifruit ETHYLENE RESPONSE FACTOR/APETALA2 (ERF/AP2)-like genes show divergence in fruit ripening associated cold and ethylene responses in C-REPEAT/DRE BINDING FACTORlike genes. PLOS ONE 14, e0216120 (2019).

45. An, J. P. et al. R2R3-MYB transcription factor MdMYB23 is involved in the cold tolerance and proanthocyanidin accumulation in apple. Plant J. 96, 562-577 (2018).

46. Zhang, A. D. et al. Transcriptome Analysis Identifies a Zinc Finger Protein Regulating Starch Degradation in Kiwifruit. Plant physiol. 178, 850-863 (2018).

47. Zeeman, S. C., Kossmann, J. \& Smith, A. M. Starch: Its Metabolism, Evolution, and Biotechnological Modification in Plants. Annu. Rev. Plant Biol. 61, 209-234 (2010).

48. Nakaminami, K. et al. Analysis of Differential Expression Patterns of mRNA and Protein During Cold-acclimation and De-acclimation in Arabidopsis. Mol. Cell. Proteom. 13, 3602-3611 (2014).

49. Zhao, Y. et al. Analysis of the cold tolerance and physiological response differences of amur grape (Vitis amurensis) germplasms during overwintering. Sci. Hortic. 259, 1-9 (2020). 108760.

50. Kaya, O. \& Kose, C. Determination of resistance to low temperatures of winter buds on lateral shoot present in Karaerik (Vitis vinifera L.) grape cultivar. Acta Physiol. Plant. 39, 1-9 (2017). 209.

51. Concetta et al. Thioredoxin-regulated $\beta$-amylase (BAM1) triggers diurnal starch degradation in guard cells, and in mesophyll cells under osmotic stress. J. Exp. Bot. 62, 545-555 (2010).

52. Yue, C. et al. Effects of cold acclimation on sugar metabolism and sugarrelated gene expression in tea plant during the winter season. Plant Mol. Biol. 88, 591-608 (2015).

53. Uemura, M. \& Steponkus, P. L. Modification of the intracellular sugar content alters the incidence of freeze-induced membrane lesions of protoplasts isolated from Arabidopsis thaliana leaves. Plant Cell Environ. 26, 1083-1096 (2010).

54. Kaplan, F. \& Guy, C. L. beta-amylase induction and the protective role of maltose during temperature shock. Plant physiol. 135, 1674-1684 (2004)

55. Moon, J. C. et al. Overexpression of Arabidopsis NADPH-dependent thioredoxin reductase $C$ (AtNTRC) confers freezing and cold shock tolerance to plants. Biochem. Bioph. Res. Co. 463, 1225-1229 (2015).

56. Huang, X. S., Wang, W., Zhang, Q. \& Liu, J. H. A basic helix-loop-helix transcription factor, PtrbHLH, of poncirus trifoliata confers cold tolerance and modulates peroxidase-mediated scavenging of hydrogen peroxide. Plant physiol. 162, 1178-1194 (2013)

57. Asada \& Kozi The water-water cycle in chloroplasts: scavenging of active oxygens and dissipation of excess photons. Annu Rev. Plant Physiol. Plant Mol. Biol. 50, 601-639 (1999).

58. Foyer, C. H. \& Shigeoka, S. Understanding oxidative stress and antioxidant functions to enhance photosynthesis. Plant physiol. 155, 93-100 (2011).

59. Wang, $X$. et al. Differential antioxidant responses to cold stress in cell suspension cultures of two subspecies of rice. Plant Cell Tiss. Org. 113, 353-361 (2013).
60. Dietz \& Karl-Josef Peroxiredoxins in plants and cyanobacteria. Antioxid. Redox Sign. 15, 1129-1159 (2011).

61. Amrina, S. et al. Simultaneous over-expression of PaSOD and RaAPX in transgenic Arabidopsis thaliana confers cold stress tolerance through increase in vascular lignifications. Plos One 9, 1-14 (2014).

62. Wei, T. et al. Enhanced ROS scavenging and sugar accumulation contribute to drought tolerance of naturally occurring autotetraploids in Poncirus trifoliata. Plant Biotechnol. J. 17, 1394-1407 (2019).

63. LV, Y. et al. The OsMYB30 transcription factor suppresses cold tolerance by interacting with a JAZ protein and suppressing beta-amylase expression. Plant physiol. 173, 1475-1491 (2017).

64. Dong, X. J. et al. The cold response regulator CBF1 promotes Arabidopsis hypocotyl growth at ambient temperatures. Embo J. 39, e103630 (2020).

65. Townley, H. E. \& Knight, M. R. Calmodulin as a potential negative regulator of Arabidopsis COR gene expression. Plant Physiol. 128, 1169-1172 (2002).

66. Li, Y. K. et al. Combined analysis of the fruit metabolome and transcriptome reveals candidate genes involved in flavonoid biosynthesis in actinidia arguta. Int. J. Mol. Sci. 19, 1-17 (2018). 1471.

67. Livak, K. J. \& Schmittgen, T. D. Analysis of relative gene expression data using real-time quantitative PCR and the 2(T)(-Delta Delta C) method. Methods $\mathbf{2 5}$, 402-408 (2001).

68. Wang, X. B. et al. PpERF3 positively regulates ABA biosynthesis by activating PpNCED2/3 transcription during fruit ripening in peach. Hortic. Res. 6, 1-10 (2019).

69. Jefferson, R. A., Kavanagh, T. A. \& Bevan, M. W. GUS fusion: beta-glucuronidase as a sensitive and versatile gene fusion marker in higher plants. Embo J. $\mathbf{6}$, 3901-3907 (1987).

70. Hellens, R. P. et al. Transient expression vectors for functional genomics, quantification of promoter activity and RNA silencing in plants. Plant Methods 1, 1-14 (2005). 13

71. Davis, A., Hall, A., Millar, A., Darrah, C. \& Davis, S. Protocol: Streamlined subprotocols for floral-DIP transformation and selection of transformants in Arabidopsis thaliana. Plant Methods 5, 1-7 (2009).

72. Ding, Y. L. et al. EGR2 phosphatase regulates OST1 kinase activity and freezing tolerance in Arabidopsis. Embo J. 38, 1-17 e99819 (2019).

73. Lappi, J. \& Luoranen, J. Testing the differences of LT 50, LD 50, or ED 50. Can. J. For. Res. 48, 729-734 (2018).

74. ThordalChristensen, H., Zhang, Z. G., Wei, Y. D. \& Collinge, D. B. Subcellular localization of $\mathrm{H}_{2} \mathrm{O} 2$ in plants. $\mathrm{H}_{2} \mathrm{O} 2$ accumulation in papillae and hypersensitive response during the barley-powdery mildew interaction. Plant J. 11, 1187-1194 (1997)

75. Jabs, T., Dietrich, R. A. \& Dangl, J. L. Initiation of runaway cell death in an Arabidopsis mutant by extracellular superoxide. Science 273, 1853-1856 (1996).

76. Su, L. Y., Dai, Z. W., Li, S. H. \& Xin, H. P. A novel system for evaluating droughtcold tolerance of grapevines using chlorophyll fluorescence. BMC Plant Biol. 15, 1-12 (2015)

77. Wang, T., Atkinson, R. \& Janssen, B. The choice of agrobacterium strain for transformation of kiwifruit. Acta Horticulturae 753, 227-232 (2007) 